\title{
SY26-2 Symposium
}

\section{Developing research projects to demonstrate the value and impact of pharmacology and clinical pharmacology}

\section{Anna Y. Zecharia}

British Pharmacological Society, UK

Focus on Pharmacology projects were developed from a set of research questions covering areas such as where pharmacology is taught, where pharmacologists work, how the discipline and related sciences should be taught in the modern world and how should graduates and trainees be supported, understanding the needs of educators and the contribution of pharmacology to the life sciences and healthcare. The work-streams were developed in parallel as external commissions, in house work by the British Pharmacological Society and external research partnerships and designed to feed into the development of the new Society strategy for 2018 onwards. This talk will give an overview of project highlights such as an audit of the education and employment landscape, development of strategic skills initiatives for in vivo sciences and clinical pharmacology and an analysis of pharmacology's role in drug discovery and development. 